\title{
High-energy phosphates metabolism and recovery in reperfused ischaemic hearts
}

\author{
M. Samaja*, S. Allibardi†, R. De Jonge $\ddagger$ \& S. L. Chierchia $\dagger$
}

*Department of Biomedical Science and Technology, University of Milan; †Scientific Institute San Raffaele, Milan, Italy; ¥Thoraxcenter, Erasmus University Rotterdam, The Netherlands

\begin{abstract}
Background The aim of this study was to assess how coronary flow, oxygen supply and energy demand affect myocardial ATP, phosphocreatine and their metabolites during oxygen shortage and recovery.

Methods Isolated rat hearts were exposed for $20 \mathrm{~min}$ to either low-flow ischaemia or hypoxaemia at the same oxygen supply, followed by return to baseline conditions $(20 \mathrm{~min})$. Seventy-three hearts were divided into four groups: ischaemic or hypoxaemic, spontaneously beating or paced to increase energy demand.
\end{abstract}

Results During $\mathrm{O}_{2}$ shortage, myocardial performance was less in ischaemic, spontaneously beating hearts (SpIs), than in the other groups (14 $\pm 1 \%$ of baseline vs. $25-48 \%$ ). Consequently, the tissue levels of ATP, total adenylates and phosphocreatine were maintained in SpIs, in contrast to marked decreases in the other groups. Upon reflow, the recovery of performance and of myocardial ATP was $94 \pm 5 \%$ in SpIs ( $P=$ NS vs. baseline) compared with $64-85 \%$ ( $P<0.05$ vs. baseline) in the other groups. The degree of recovery was positively related to the ischaemic contents of ATP $(P=0.03)$ and adenylates $(P=0 \cdot 001)$, but not to that of phosphocreatine $(P=\mathrm{NS})$.

Conclusion The maintenance of the ATP pool under low oxygen supply conditions is essential for good recovery. The most important factors that determine the ATP pool size are the energy demand, which increases the formation of diffusible ATP catabolites, and the coronary flow, which removes these catabolites, rather than the oxygen supply per se.

Keywords ATP, energy demand, hypoxia, myocardial metabolism, phosphocreatine, purine metabolism.

Eur F Clin Invest 1998: 28 (12): 983-988

\section{Introduction}

Although the only way to recover ischaemic hearts, reflow is often associated with damage. Only part of this damage, however, is due to the free radicals generated concomitantly with oxygen readmission [1]. The biochemical mechanisms underlying damage are still elusive, but highenergy phosphates are probably involved, because damage is limited if the tissue ATP level is maintained during ischaemia [2-4]. The role of ongoing glycolysis is controversial, as it appears detrimental during no-flow ischaemia but beneficial during low-flow ischaemia [5]. However, the implications of glycolysis, especially in relation to ischaemic preconditioning, are described elsewhere [6].

Correspondence: Dr M. Samaja, Dipartimento di Scienze e Tecnologie Biomediche, LITA, via F.Cervi 93, 20090 Segrate Milan, Italy. E-mail Michele.Samaja@unimi.it

Received 19 September 1997; accepted 17 July 1998
Assessing the effects of energy demand and coronary flow on the metabolism of total adenine nucleotides and purines (TANP) may help to clarify the above issue. We have recently shown that a situation of low coronary flow, by depressing the washout of lactate, can strongly downregulate myocardial performance $[7,8]$. In addition, coronary flow appears crucial in determining recovery after ischaemia as it helps to wash out diffusible TANP, i.e. inosine, hypoxanthine, xanthine and urate, thereby reducing tissue ATP level [9]. Although it has already been hypothesized that residual flow increases the washout of TANP formed during $\mathrm{O}_{2}$ shortage $[10,11]$, the relationship between reperfusion injury, tissue TANP, coronary flow and energy demand has not yet been established.

In this work, we verify the existence of such link by testing the hypothesis that dysfunction measured during reflow depends on factors which depend on both the energy demand and the residual coronary flow during ischaemia. High energy demand enhances formation of ATP-derived 
membrane-diffusible substances, whereas high residual coronary flow promotes definitive loss of these substances for the contractile system. By comparing various experimental conditions characterized by the same $\mathrm{O}_{2}$ supply yet different energy demand and coronary flow levels during ischaemia, we show that the maintenance of the ATP level during $\mathrm{O}_{2}$ shortage protects hearts. In addition, we show that both high energy demand and high coronary flow can determine the size of the ATP pool during the recovery phase.

\section{Materials and methods}

\section{Heart perfusion}

Ad libitum-fed male Sprague-Dawley rats (250-280g) were anaesthetized by i.p. heparinized sodium thiopental $\left(10 \mathrm{mg} 100 \mathrm{~g}^{-1}\right.$ b.w.); hearts were excised and perfused at $37^{\circ} \mathrm{C}$ with a medium containing (in $\mathrm{mmol} \mathrm{L}^{-1}$ ) $\mathrm{NaCl}$ $(115 \cdot 6), \mathrm{KCl}(4 \cdot 7) \mathrm{KH}_{2} \mathrm{PO}_{4}(1 \cdot 2)$, EDTA (0.5), $\mathrm{Na}_{2} \mathrm{SO}_{4}$ $(1 \cdot 2), \mathrm{NaHCO}_{3}(28 \cdot 5), \mathrm{CaCl}_{2}(2 \cdot 5), \mathrm{MgCl}_{2}(1 \cdot 2)$ and glucose (16.6), $\mathrm{pH} 7 \cdot 40 \pm 0 \cdot 02$ at $\mathrm{PCO}_{2}=43 \mathrm{mmHg}$. A roller pump (Gilson, France) delivered the medium at preselected flows to a filter $(8 \mu \mathrm{m}$ pore size, $47 \mathrm{~mm}$ diameter, Nuclepore Pleasanton, CA, USA), a membrane oxygenator, flowed with gas at $\mathrm{PO}_{2}$ of either 670 or 67 $\mathrm{mmHg}$, a preheater and the aortic cannula. The venous return was collected from the pulmonary artery for measurement of venous $\mathrm{PO}_{2}$ (YSI model 5300 Oxygen Monitor, Yellow Springs, OH, USA) and $\mathrm{O}_{2}$ uptake $\left(V_{\mathrm{O}_{2}}\right)$. A saline-filled balloon in the left ventricle was connected to a pressure transducer (Harvard Apparatus model 52-9966, Natick, MA, USA) for measurement of end-diastolic pressure (EDP), left-ventricle developed pressure (LVDP) and heart rate (HR). Hearts from the paced groups were stimulated throughout at $330 \mathrm{~min}^{-1}$ by electrodes (Harvard, South Natick, MA, USA: square wave stimulator, $5 \mathrm{~ms}$ pulse duration, $10 \mathrm{~V}$ pulse amplitude) placed on the aortic cannula and on the apex of the ventricle. To account for different $H R s$ in the various groups, myocardial performance is expressed as $\mathrm{LVDP} \times \mathrm{HR}$.

\section{Experimental protocol}

Hearts were stabilized for $30 \mathrm{~min}$ at flow $=15 \mathrm{~mL} \mathrm{~min}^{-1}$,

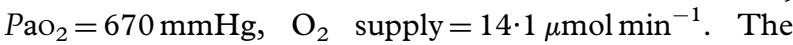
balloon volume was adjusted to achieve an EDP of approximately $10 \mathrm{mmHg}$ and was then kept constant throughout. Hearts were assigned to one of the following groups: SpIs (spontaneously beating hearts exposed to low-flow ischemia and then reperfused at a flow rate of $15 \mathrm{~mL} \mathrm{~min}^{-1}$; $n=9$ ); SpHy (spontaneously beating hearts exposed to hypoxaemia and then reoxygenated at $\mathrm{PaO}_{2}=670 \mathrm{mmHg}$; $n=8$ ); PxIs (paced hearts exposed to low-flow ischaemia and then reperfused at a flow rate of $\left.15 \mathrm{~mL} \mathrm{~min}^{-1} ; n=9\right)$; PxHy (paced hearts exposed to hypoxaemia and then reoxygenated at $\mathrm{PaO}_{2}=670 \mathrm{mmHg} ; n=8$ ). Recovery was quantitated as the ratio (performance during reflow or reoxygenation)/(performance during baseline). Low-flow ischaemia and hypoxaemia were maintained for $20 \mathrm{~min}$ either by reducing flow to $1.5 \mathrm{~mL} \mathrm{~min}^{-1}$ or by switching to the low $-\mathrm{PO}_{2}$ gas. The two conditions were thus matched for $\mathrm{O}_{2}$ supply ( $10 \%$ of baseline), duration (20 min) and temperature $\left(37^{\circ} \mathrm{C}\right)$. Hearts were freeze-clamped at the end of reflow or reoxygenation $(20 \mathrm{~min})$ to determine tissue content of high-energy phosphates and their metabolites.

In parallel experiments, additional hearts were freezeclamped after the periods of baseline (seven spontaneously beating and six placed), low-flow ischaemia (7/6) and hypoxaemia $(8 / 5)$.

\section{High performance liquid chromatography}

Hearts were freeze-clamped with aluminium clamps and then cooled in liquid nitrogen; tissue was extracted with 0.5 mol $\mathrm{L}^{-1}$ perchloric acid, neutralized and analysed for ATP, ADP, AMP, adenosine, inosine-5'-monophosphate, inosine, hypoxanthine, xanthine, urate, creatine and phosphocreatine (Pcr) by high-performance liquid chromatography as previously described [12]. Using this technique, hypoxanthine coelutes with xanthine and inosine with urate. The level of TANPs was calculated as the sum of all the above substances except creatine and Pcr. The diffusible substances include adenosine, inosine, hypoxanthine, xanthine and urate.

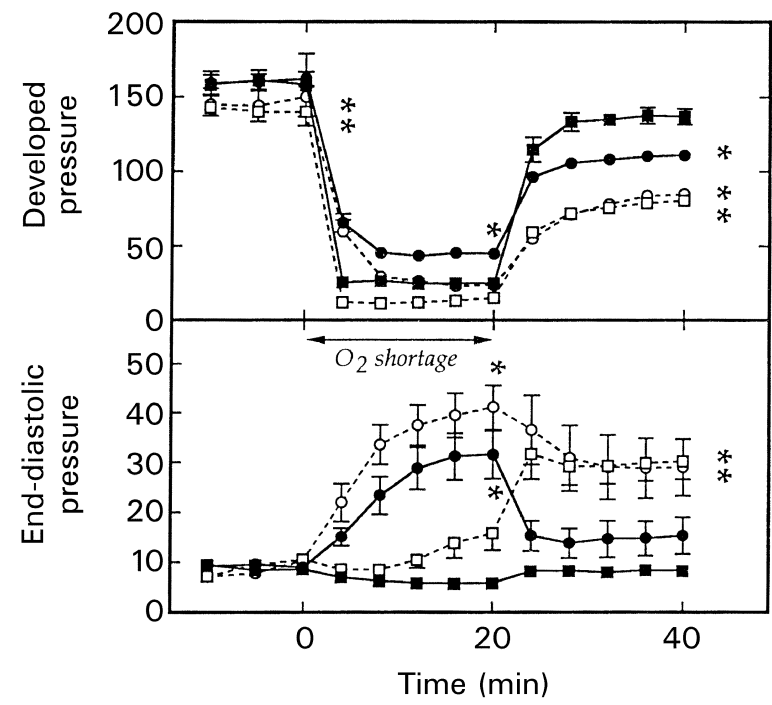

Figure 1 Developed and end-diastolic pressures $(\mathrm{mmHg})$. The $\mathrm{O}_{2}$ supply was reduced at $t=0-20 \mathrm{~min}$ by decreasing either coronary flow or $\mathrm{PO}_{2}$ to $10 \%$ of baseline. The vertical bars represent SE. Two-way ANova for both, $P<0 \cdot 0001$. SpIs ( $\square$ ), spontaneously beating hearts exposed to low-flow ischaemia and reflow; $\mathrm{SpHy}(\bullet)$, spontaneously beating hearts exposed to hypoxaemia and reoxygenation; PxIs $(\square)$, paced hearts exposed to low-flow ischaemia and reflow; $\mathrm{PxHy}(\mathrm{O})$, paced hearts exposed to hypoxaemia and reoxygenation. *Significant difference vs. SpIs. 

$\times$ heart rate

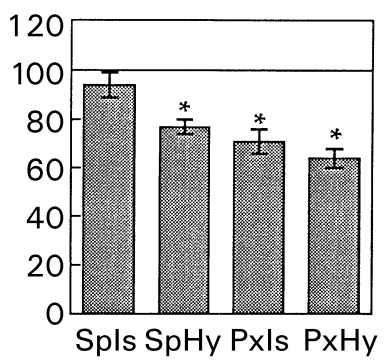

Developed pressure
$\mathrm{O}_{2}$ uptake

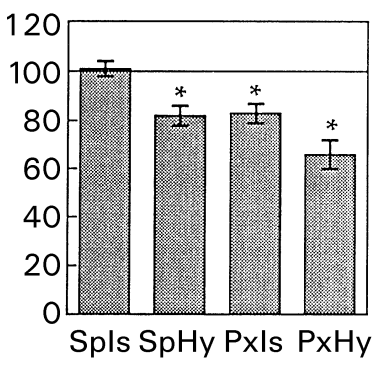

Figure 2 Performance recovery at end of reflow or reoxygenation. Data are expressed as per cent of baseline values. Two-way ANOva, $P<0.0001$ and $P=0.08$ for developed pressure $\times$ heart rate and $V_{\mathrm{o}_{2}}$ respectively. Same conventions as in Fig. 1.

\section{Statistics}

Data are expressed as means \pm SE. To detect interactions between the various groups and three consecutive perfusion conditions (baseline, $\mathrm{O}_{2}$ shortage, and recovery), we used to analysis of variance (ANOVA) procedures (StatView, Abacus Concepts, Berkeley, CA, USA), depending on the variable being tested: two-way, repeated-measures ANOvA for variables available for each heart under the three conditions; and factorial, six-groups ANOvA for variables such as, for example, the myocardial metabolites content. In both cases, if the ANOva test was significant $(P<0.05)$, hearts were compared with the Bonferroni/Dunnett multiple comparison procedure.

\section{Results}

\section{General}

Hearts did not stop contracting during $\mathrm{O}_{2}$ shortage and recovery; thus, their performance could be monitored continuously (Fig. 1). Figures 1 and 2 show that, although almost complete in SpIs, functional recovery upon reflow or reoxygenation was impaired in the other groups. Figures 3 and 4 report the tissue metabolite levels measured at the end of the various phases. As observed for myocardial performance, the recovery of ATP and TANP was almost complete in SpIs, in contrast to progressive impairment in the other groups. However, both Pcr and the sum Pcr + creatine were maintained in all the groups. Electron microscopy did not reveal signs of irreversible injury in any group (not shown).

\section{Effects of pacing at full $\mathrm{O}_{2}$ supply (baseline)}

LVDP was lower in paced than in spontaneously beating hearts, with higher $V_{\mathrm{O}_{2}}$ (Table 1). However, HR was higher in paced hearts, so that LVDP $\times$ HR was the same in the two groups. No differences were observed for EDP or most metabolic parameters, with the exception of inosine-5'monophosphate, which was higher, and adenosine, which was lower in paced than in spontaneously beating hearts.

\section{Low-flow ischaemia and reflow in spontaneously beating hearts (SpIs)}

As expected myocardial contractility decreased abruptly at the onset of low-flow ischaemia. In addition, HR decreased to $178 \pm 12 \mathrm{~min}^{-1}(P<0.0001$ with respect to baseline). However, there were no signs of diastolic contracture, and the level of tissue metabolites was preserved throughout the ischaemic period. At the end of the reflow, none of the examined parameters, including $\operatorname{HR}\left(264 \pm 15 \mathrm{~min}^{-1}\right)$, was significantly different from baseline. This indicates near complete functional and metabolic recovery in SpIs.
Figure 3 Myocardial content of adenine nucleotides and purines in spontaneously contracting (left) and paced (right) hearts. The bars on the top represent IMP on a different scale range. One-way ANOva, $P<0 \cdot 0001$ for all substances. ${ }^{\#}$ Significant difference $(P<0.05)$ in the toal content of adenine nucleotides and purines compared with spontaneously beating hearts exposed to low-flow ischaemia and reflow (SpIs). *Significant difference $(P<0.05)$ compared with spontaneously beating hearts exposed to low-flow ischaemia and reflow (SpIs).

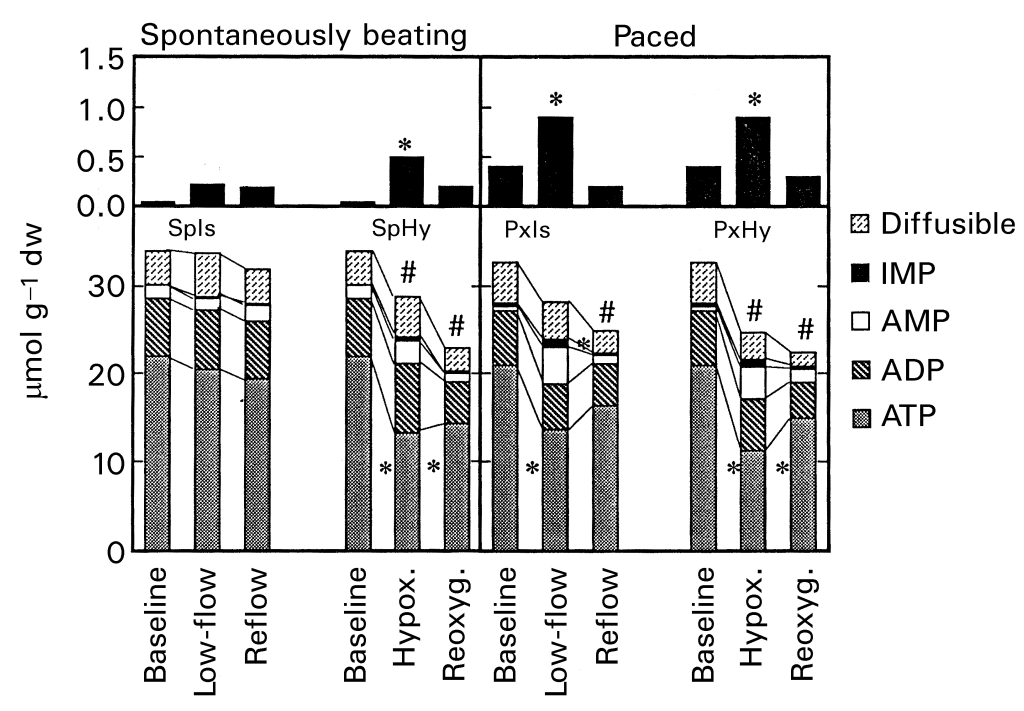




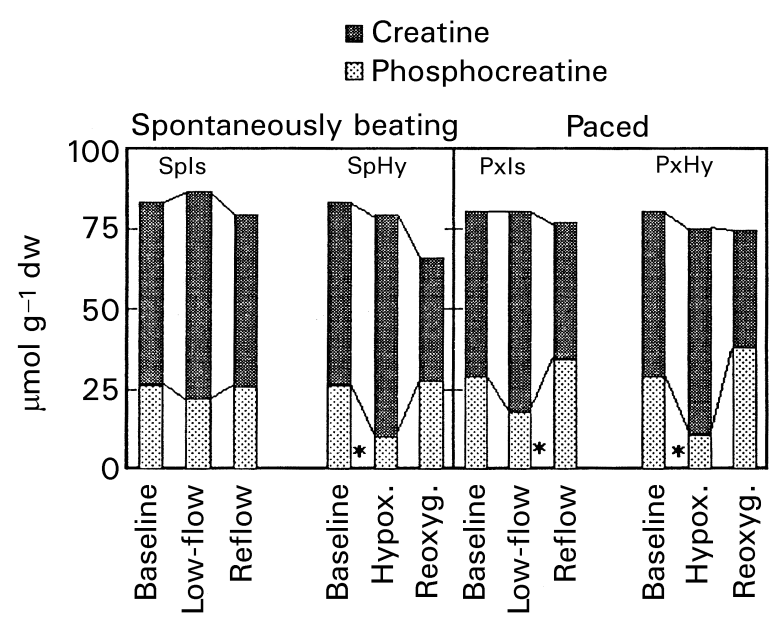

Figure 4 Myocardial content of creatine and phosphocreatine. One-way anova $P<0.0001$ for all substances. Same conventions as in Fig. 3.

\section{Effects of coronary flow (SpHy)}

In $\mathrm{SpHy}$ the decrease of myocardial contractility at the onset of $\mathrm{O}_{2}$ shortage was less pronounced than in SpIs. Thus, the myocardial performance was higher in $\mathrm{SpHy}$ than in SpIs even at the same supply of $\mathrm{O}_{2}$. In addition, EDP was higher, indicating onset of diastolic contracture. The levels of ATP, Pcr and TANP were lower, with higher IMP. Upon return to baseline conditions, HR recovered completely $\left(261 \pm 17 \mathrm{~min}^{-1}, P=\mathrm{NS}\right.$ with respect to baseline), but LVDP, LVDP $\times$ HR, ATP and TANP were lower than during baseline, with higher EDP. This indicates consistent damage in SpIs.

\section{Effects of high energy demand (PxIs)}

During low-flow ischaemia, PxIs developed significant diastolic contracture. Although LVDP was similar to that of SpIs the total work output $(\mathrm{EDP}+\mathrm{LVDP}) \times \mathrm{HR}$ was
$9 \cdot 9 \pm 0.7$ in PxIs vs. $6 \cdot 2 \pm 0.5 \mathrm{mmHg} \times 10^{3} \mathrm{~min}^{-1}$ in SpIs $(P<0 \cdot 01)$. This indicates that PxIs performed at higher level than SpIs. At the end of the reflow, the recovery of LVDP $\times$ HR, tissue ATP and TANP was superimposable on that observed for $\mathrm{SpHy}$.

\section{Simultaneous effects of high energy demand and coronary flow (PxHy)}

During $\mathrm{O}_{2}$ shortage, PxHy developed a pattern superimposable on that of PxIs and SpHy: high EDP and LVDP with lower ATP, TANP and Pcr. During reoxygenation, the recovery of LVDP, LVDP $\times \mathrm{HR}$, ATP and TANP was also similar to that observed in $\mathrm{SpHy}$ and PxIs.

\section{Discussion}

The design of this study allowed us to assess separately the effects of residual coronary flow by comparing SpIs with SpHy, and those of energy demand by comparing SpIs with PxIs. Group PxHy assessment of the simultaneous effects of coronary flow and energy demand. The product LVDP $\times$ $\mathrm{HR}$ is an index of myocardial function in this model because it is not affected by pacing in normoxic hearts. The higher $V_{\mathrm{O}_{2}}$ in paced hearts might be due to greater $\mathrm{O}_{2}$ cost of increasing HR compared with force development.

\section{Bioenergetics of low-flow ischaemia and hypoxaemia}

In spontaneously beating hearts, lowflow ischaemia downregulates myocardial performance to a much greater extent than hypoxaemia. Identifying the factors that determine down-regulation is beyond the aims of this study, but presumably different degrees of lactate or $\mathrm{H}^{+}$washout secondary to different coronary flow rates are involved

Table 1 Myocardial performance and metabolism (mean \pm SE) at baseline.

\begin{tabular}{|c|c|c|c|}
\hline & Spontaneous & $P$ & Paced \\
\hline$n$ & 39 & & 34 \\
\hline $\operatorname{HR}\left(\min ^{-1}\right)$ & $266 \pm 6$ & $<0 \cdot 0001$ & $330 \pm 0$ \\
\hline $\mathrm{EDP}(\mathrm{mmHg})$ & $9 \cdot 4 \pm 0 \cdot 5$ & NS & $9 \cdot 8 \pm 0 \cdot 3$ \\
\hline LVDP $(\mathrm{mmHg})$ & $148 \pm 3$ & $<0 \cdot 0001$ & $115 \pm 3$ \\
\hline $\operatorname{LVDP} \times \operatorname{HR}\left(\mathrm{mmHgx} 10^{3} \mathrm{~min}-1\right)$ & $39 \cdot 4 \pm 1 \cdot 2$ & NS & $37 \cdot 9 \pm 1 \cdot 0$ \\
\hline$V \mathrm{O}_{2}\left(\mu \mathrm{mol} \mathrm{min}{ }^{-1}\right)$ & $6 \cdot 68 \pm 0 \cdot 40$ & $0 \cdot 008$ & $7 \cdot 95 \pm 0 \cdot 20$ \\
\hline $\operatorname{ATP}\left(\mu \mathrm{molg}^{-1}\right.$ dry wt $)$ & $21 \cdot 9 \pm 1 \cdot 6$ & NS & $20 \cdot 9 \pm 0 \cdot 7$ \\
\hline $\mathrm{IMP}\left(\mu \mathrm{molg}^{-1}\right.$ dry wt $)$ & $0.04 \pm 0.04$ & $0 \cdot 001$ & $0 \cdot 36 \pm 0 \cdot 04$ \\
\hline Adenosine $\left(\mu \mathrm{mol} \mathrm{g}^{-1}\right.$ dry wt $)$ & $0 \cdot 54 \pm 0 \cdot 15$ & $0 \cdot 01$ & $0 \cdot 15 \pm 0 \cdot 07$ \\
\hline TANP $\left(\mu \mathrm{mol} \mathrm{g}^{-1}\right.$ dry wt $)$ & $33 \cdot 9 \pm 2 \cdot 6$ & NS & $32 \cdot 6 \pm 1 \cdot 1$ \\
\hline $\operatorname{Pcr}\left(\mu \mathrm{molg}^{-1}\right.$ dry wt $)$ & $26 \cdot 4 \pm 2 \cdot 4$ & NS & $27 \cdot 7 \pm 2 \cdot 5$ \\
\hline
\end{tabular}

The differences between the two groups were tested by Student's $t$-test for unpaired samples. For metabolites, $n=7$ and 6 for spontaneous and paced hearts respectively. IMP, inosine- $5^{\prime}$ monophosphate. 


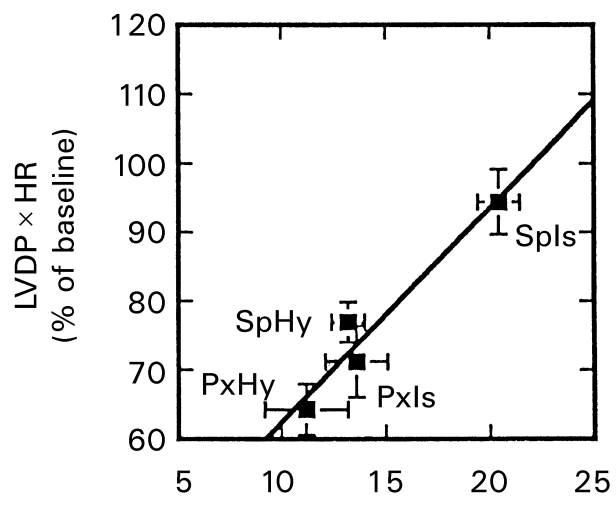

ATP

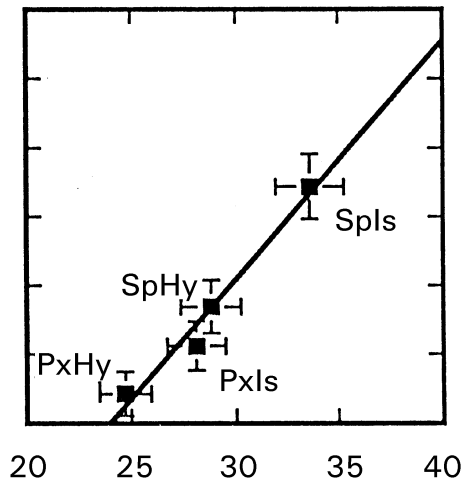

Total adenine nucleotides and purines

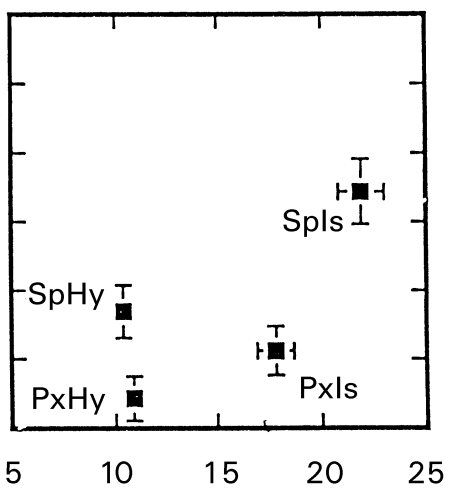

Phosphocreatine

$\mu \mathrm{mol} \mathrm{g}^{-1} \mathrm{dw}$

Figure 5 Relationship between per cent LVDP $\times$ HR recovery and the myocardial contents of ATP, sum of adenine nucleotides and purines or phosphocreatine, measured at the end of $\mathrm{O}_{2}$ shortage. Best-fit lines are: ATP, $y=31 \cdot 21+3 \cdot 12 x, r^{2}=0 \cdot 92$, $P=0 \cdot 05$; for TANP, $y=-23 \cdot 1+3 \cdot 44 x, r^{2}=0 \cdot 98, P=0 \cdot 005$; for

[7]. By reducing contractility and by maintaining the levels of ATP, TANP and Pcr near preischaemic values, SpIs achieve a favourable perfusion-contraction matching between energy supply and demand, which is compatible with short-term hibernation [13]. This allows greater recovery in these hearts. Both high flow and pacing unmatch the perfusion-contraction matching by increasing energy demand. This results in a fall in high-energy phosphates, increased ATP catabolism, increased washout of diffusible substances and impaired recovery following return to baseline conditions.

The fact that oxidative metabolism $\left(\mathrm{VO}_{2}\right)$ during $\mathrm{O}_{2}$ shortage was the same in all groups stresses the increasing importance of glycolytic ATP, which meets the energy requirements under $\mathrm{O}_{2}$ shortage conditions. Presumably, endogenous glycogen is the most important fuel source to sustain the higher contractility in SpHy [8]. We did not measure lactate production in PxIs and PxHy but the data reported in [7] show that pacing increases the rate of glycolysis in ischaemic hearts only, whereas this rate is unaffected in hypoxaemic hearts. Perhaps, in PxIs and $\mathrm{SpHy}$, the glycolytic rate is already near maximum. In fact, the values of lactate production during hypoxaemia $\left(9 \cdot 2-10 \cdot 8 \mu \mathrm{mol} \mathrm{min}^{-1} \mathrm{~g}^{-1}\right.$ wet wt or $71-83 \mu \mathrm{mol} \mathrm{min}^{-1}$ $\mathrm{g}^{-1}$ dry wt) are close to the values of $10-14 \mu \mathrm{mol} \mathrm{min}^{-1} \mathrm{~g}^{-1}$ wet wt [14] and 60-70 $\mu \mathrm{mol} \mathrm{min}^{-1} \mathrm{~g}^{-1}$ dry wt [15] reported for contracting anoxic isolated rate hearts.

Although its role in heart muscle is not yet fully clear, IMP is considered a good index of metabolic derangement during $\mathrm{O}_{2}$ shortage. In addition, the lowest IMP level during $\mathrm{O}_{2}$ shortage (SpIs) was associated with the greatest down-regulation of myocardial performance during ischaemia. Photoaffinity labelling studies in rat have revealed the presence of IMP binding sites on the myosin complex [16]. phosphocreatine, $r^{2}=0 \cdot 50, P=\mathrm{NS}$. SpIs, spontaneously beating hearts exposed to low-flow ischaemia and reflow; SpHy, spontaneously beating hearts exposed to hypoxaemia and reoxygenation; PxIs, paced hearts exposed to low-flow ischaemia and reflow; $\mathrm{PxHy}$, paced hearts exposed to hypoxaemia and reoxygenation.

In addition, IMP inhibits actin-activated $\mathrm{Mg}^{2+}$-myosin ATPase activity [17]. Finally, loss of performance in rat medial gastrocnemius muscle is associated with increases in muscle IMP content [18]. In the normoxic heart, AMP is mainly deaminated to IMP because AMP deaminase activity is much larger than that of $5^{\prime}$-nucleotidase. However, during $\mathrm{O}_{2}$ shortage, increases in $\mathrm{P}_{\mathrm{i}}$ inhibit AMP deaminase, resulting in the release of adenosine $[19,20]$.

\section{Diffusible substances and myocardial dysfunction}

Loss of diffusible substances is crucial to determine recovery in this model. In oxygenated tissues, ATP dephosphorylation is tightly coupled with ADP phosphorylation; thus, the loss of diffusible ATP catabolites is slow and compensated by purine salvage and de novo synthesis [21]. When energy supply is low relative to demand, ATP breakdown may exceed synthesis, thereby increasing the amount of diffusible catabolites. This does not occur in SpIs because of perfusion-contraction matching, but TANP progressively decreases in the other groups. Thus, both the excessive ATP breakdown with respect to synthesis and the high coronary flow finally lead to loss of diffusible substances. TANP loss is not accompanied by parallel changes in Pcr + creatine because both Pcr and creatine do not leak across intact membranes [22]. Therefore, TANP depletion is a metabolic phenomenon that occurs even in the absence of irreversible membrane damage. This is confirmed by lack of ultrastructural alterations and of enzyme release. The preservation of the ATP pool during $\mathrm{O}_{2}$ shortage is a major contributor to heart protection (Fig. 5), in agreement with previous studies $[2,3,23]$. 


\section{Implications of this study}

A relationship between functional recovery and maintenance of diffusible substances has emerged. In this model, low coronary flow and low energy demand maintain diffusible substances homeostasis. In contrast, the $\mathrm{O}_{2}$ supply per se does not appear to be as critical. High coronary flow and high energy demand increase the amount of diffusible substances formed from ATP catabolism, result in low tissue ATP levels and are associated with poor recovery. The extent of recovery critically depends on both energy demand and flow during ischaemia, and not on the severity of $\mathrm{O}_{2}$ shortage.

\section{Acknowledgements}

We gratefully thank Professor J. W. deJong for helpful advice and encouragement.

\section{References}

1 Samaja M, Motterlini R, Santoro F, Dell'Antonio G, Corno A. Oxidative injury in reoxygenated and reperfused hearts. Free Rad Biol Med 1994;16:255-62.

2 Haas GS, DeBoer LWV, O'Keefe DDO, Bodenhamer RM, Geffin GA, Drop LJ et al. Reduction of postischemic myocardial dysfunction by substrate repletion during reperfusion. Circulation 1984;70:65-74.

3 Rubin BB, Liauw S, Tittley J, Romaschin AD, Walker PM. Prolonged adenine nucleotide resynthesis and reperfusion injury in postischemic skeletal muscle. Am J Physiol 1992; 262:H1538-47.

4 Schaefer S, Carr LJ, Prussel E, Ramasamy R. Effects of glycogen depletion on ischemic injury in isolated rat hearts: insights into preconditioning. Am J Physiol 1995;268:H93544.

5 de Jonge R, Bradamante S, Speleman L, de Jong JW. Carbohydrates and purines in underperfused hearts protected by ischemic preconditioning. J Mol Cell Cardiol 1998;30:699708 .

6 de Jong JW, de Jonge R, Marchesani A, Janssen M, Bradamante S. Controversies in preconditioning. Cardiovasc Drug Ther 1996;10:767-73.

7 Samaja M, Casalini S, Allibardi S, Corno A, Chierchia S. Regulation of bioenergetics in $\mathrm{O}_{2}$-limited isolated rat hearts. J Appl Physiol 1994;77:2530-6.

8 Merati G, Allibardi S, Monti LD, de Jong JW, Samaja M. Dynamics of myocardial adaptation to low-flow ischemia and hypoxemia. Am J Physiol 1996;271:2300-5.
9 Samaja M, Motterlini R, Allibardi S, Casalini S, Merati G, Corno A, et al. Myocardial metabolism and function in acutely ischemic and hypoxemic isolated rat hearts. J Mol Cell Cardiol 1995;27:1213-18.

10 Bak MI, Ingwall JS. Acidosis during ischemia promotes adenosite triphosphate resynthesis in postischemic rat heart. J Clin Invest 1994;93:40-49.

11 Soussi B, Lagerwall K, Idstrom JP, Schersten T. Purine metabolic pathways in rat hindlimb perfusion model during ischemia and reperfusion. Am J Physiol 1993;265:H1074-81.

12 Motterlini R, Samaja M, Tarantola M, Micheletti R, Bianchi G. Functional and metabolic effects of propionyl-L-cartinine in the isolated perfused hypertrophied rat heart. Mol Cell Biochem 1992;116:139-45.

13 Ross J. Myocardial perfusion-contraction matching. Implications for coronary heart disease and hibernation. Circulation 1991;83:1076-83.

14 Matthews PM, Taylor DJ, Radda GK. Biochemical mechanisms of acute contracture failure in the hypoxic rat heart. Cardiovasc Res 1986;20:13-19.

15 Williamson JR. Glycolytic control mechanisms. II. Kinetics of intermediate changes during the aerobic-anoxic transition in perfused rat heart. J Biol Chem 1966;241:5026-36.

16 Berden JA, de Haan A, de Haan EJ, van Doorn JE, Hartog $\mathrm{AF}$, Westra HG. Has IMP a regulatory role during fatiguing contraction? IMP-binding sites on the myosin complex of rat muscle. J Physiol 1986;381:85P (Abstract).

17 Westra HG, Berden JA, Pasman WJ. A model for the regulation of actin-activated $\mathrm{Mg}^{2+}$-myosin ATPase activity: inhibition of the formation of actin-myosin complex by IMP. In: Sargeant AJ, Kernell D, editor. Neuromuscular fatigue. Amsterdam: Royal Netherlands Academy of Sciences, Elsevier Biomedical; 1997: p.24-6.

18 de Haan A. High-energy phosphates and fatigue during repeated dynamic contractions of rat muscle. Exp Physiol 1990:75:851-4.

19 Chen W, Hoerter J, GuTetharon M. AMP degradation in the perfused rat heart during 2-deoxy-D-glucose perfusion and anoxia. The release of adenosine and inosine. J Mol Cell Cardiol 1996;28:2163-74.

20 Chen W, GuTetharon M. AMP degradation in the perfused rat heart during 2-deoxy-D-glucose perfusion and anoxia. I. The determination of the degradation pathways using an adenosine deaminase inhibitor. J Mol Cell Cardiol 1996;28: 2175-82.

21 Zimmer HG, Trendelenburg C, Kammermeier H, Gerlach E. De novo synthesis of myocardial adenine nucleotides in the rat. Circ Res 1973;32:635-42.

22 Reimer KA, Jennings RB, Hill ML. Total ischemia in dog hearts, in vitro. 2 . High energy phosphate depletion and associated defects in energy metabolism, cell volume regulation, and sarcolemmal integrity. Circ Res 1981;49:901-11.

23 Takeo S, Tanonaka K, Miyake K, Imago M. Adenine nucleotides metabolites are beneficial for recovery of cardiac contractile force after hypoxia. J Mol Cell Cardiol 1988;20: 187-99. 